\title{
SUB-SYNCHRONOUS VIBRATIONS OF A ROTOR SUPPORTED ON POORLY LUBRICATED JOURNAL BEARINGS
}

\author{
L. Smolík* ${ }^{*}$ P. Polach ${ }^{* *}$, J. Rendl ${ }^{* * *}$
}

\begin{abstract}
This paper aims to the modelling and investigation of sub-synchronous vibrations of a rotor supported on poorly lubricated journal bearings. Bores for an oil supply are located in the lower halves of bearing shells. It is shown that the poor lubrication of the described bearings may lead to an occurrence of stable vibrations at the frequency ca. 0.9 to the rotor speed. Simulations are performed using a multi-body approach for modelling rotating systems. Forces acting in journal bearings are evaluated employing a massconserving finite element solution of the Reynolds equation.
\end{abstract}

Keywords: Rotor dynamics, Journal bearings, Sub-synchronous vibrations, Poor lubrication.

\section{Introduction}

Conditions which lead to an occurrence of sub-synchronous vibrations (SSVs) of rotors supported on poorly lubricated journal bearings are reported in this paper. The reported SSVs are at the frequency ca. 0.9 to the rotor speed. This frequency does not correspond with the frequency of self-excited vibrations due to oil-whirl and oil-whip instabilities (Bently \& Hatch, 2003; Muszynska, 2005) nor with any natural frequency of the rotating system. However, there might be a connection between the reported SSVs and the fluid-induced instability - both Someya (1989, p 301) and Muszynska (2005, pp 236-242) noted that poor lubrication has an influence on the unstable vibrations due to the fluid-induced instability.

Similar vibrational patterns were observed also by DeCamillo (2006) in thrust bearings. DeCamillo noted that these SSVs usually occur in poorly lubricated bearings but he was unable to identify all conditions which can lead to the reported SSVs. The problem of these SSVs is not commonly studied in available literature and, therefore, the investigation of this problem is a challenge.

The computational analysis was performed via multi-body approach for modelling which is described in Section 2. The calculations of pressure distribution in journal bearings were performed employing a massconserving finite element solution of the Reynolds equation. Thus, hydrodynamic forces acting in the journal bearings are non-linear. This phenomenon is investigated on the computational model of assembled experimental testing rig that enables investigation of fluid-induced instabilities in journal bearings whose parameters are discussed in Section 3. The results are introduced and discussed in Section 4 and the matter is briefly summarized at the end of this paper.

\section{Equations of motion of a flexible rotor supported on journal bearings}

The motion of the flexible rotor is described employing a multi-body formalism. Motions of the rotor are decomposed to elastic and gross coordinates. In order to simulate both types of the motion, the system of

\footnotetext{
Ing. Luboš Smolík: New Technologies for the Information Society, European Centre of Excellence, University of West Bohemia, Univerzitní 8; 306 14, Plzeň; CZ, carlist@ntis.zcu.cz

** Dr. Ing. Pavel Polach: New Technologies for the Information Society, European Centre of Excellence, University of West Bohemia, Univerzitní 8; 306 14, Plzeň; CZ, ppolach@ntis.zcu.cz

*** Ing. Jan Rendl: New Technologies for the Information Society, European Centre of Excellence, University of West Bohemia, Univerzitní 8; 306 14, Plzeň; CZ, rendlj@ntis.zcu.cz
} 
differential algebraic equations described by Lorenz et al. (2017) is employed (time $t$ is omitted):

$$
\begin{gathered}
\mathbf{M} \ddot{\mathbf{q}}+\mathbf{f}^{r b}(\mathbf{z}, \dot{\mathbf{z}})=\mathbf{f}^{g y r}(\mathbf{z})+\mathbf{f}^{j}(\mathbf{z}, \mathbf{w})+\mathbf{f}^{e}(\mathbf{z})-\mathbf{D} \dot{\mathbf{q}}-\mathbf{K} \mathbf{q}, \\
\mathbf{S}\left(\boldsymbol{\theta}_{B}\right) \dot{\boldsymbol{\theta}}_{B}=\mathbf{\Omega}_{B}, \\
\boldsymbol{\theta}_{B}^{\top} \boldsymbol{\theta}_{B}=1, \\
r(\mathbf{q})=0 .
\end{gathered}
$$

The position, orientation and deformation of the rotor are included in state vector $\mathbf{z}^{\top}=$ $\left[\mathbf{x}_{B}^{\top}, \boldsymbol{\theta}_{B}^{\top}, \dot{\mathbf{x}}_{B}^{\top}, \boldsymbol{\Omega}_{B}^{\top}, \mathbf{q}^{\top}, \dot{\mathbf{q}}^{\top}\right]$. Vector $\mathbf{x}_{B} \in \mathbb{R}^{3}$ and quaternion $\boldsymbol{\theta}_{B} \in \mathbb{R}^{4}$ characterise the position and the orientation of the rotor and vector $\mathbf{q} \in \mathbb{R}^{N}$ contains elastic coordinates relative to a coordinate system which is defined by $\mathbf{x}_{B}$ and $\boldsymbol{\theta}_{B}$. Vector $\mathbf{w}$ is composed of state vectors of all bodies coupled to the rotor. $\mathbf{M}, \mathbf{D}, \mathbf{K}$ are the mass, damping and stiffness matrix, respectively. $\mathbf{f}^{r b} \in \mathbb{R}^{6 N}, \mathbf{f}^{g y r} \in \mathbb{R}^{6 N}$ are vectors of forces which result from rigid body accelerations and gyroscopic effects, respectively (Offner et al., 2006). Vector $\mathbf{f}^{j} \in \mathbb{R}^{6 N}$ accommodate forces in couplings and $\mathbf{f}^{e} \in \mathbb{R}^{6 N}$ prescribes external loading.

Equation (2) describes the relation between angular velocity $\boldsymbol{\Omega}_{B}$ of the gross motion and quaternion $\boldsymbol{\theta}_{B}$, Equation (3) is the normalization condition and Equation (4) is introduced in order to obtain a unique separation of the gross and elastic coordinates. Equation (4) is discussed in detail by Offner et al. (2006).

The most prominent coupling forces act on the rotor in its bearings. These so-called hydrodynamic forces can be obtained by integrating a pressure in an oil film over the surface of the bearing. We assume that the oil is an incompressible Newtonian fluid, the film is thin, the flow in the film is laminar and cavitation may occur. Pressure $p$ is then governed by the Reynolds equation in the form (Lorenz et al., 2017):

$$
\frac{\partial}{\partial s}\left(\frac{\theta h^{3}}{12 \mu} \frac{\partial p}{\partial s}\right)+\frac{\partial}{\partial x}\left(\frac{\theta h^{3}}{12 \mu} \frac{\partial p}{\partial x}\right)=\frac{u_{j}+u_{s}}{2} \frac{\partial(\theta h)}{\partial s}+\frac{\partial(\theta h)}{\partial t},
$$

where $s, x$ are the circular and the axial coordinates, respectively, $h$ is the gap between a journal and a shell, $\theta$ is the percentage of $h$ which is filled with oil, $\mu$ is the dynamic viscosity of the oil and $u_{j}, u_{s}$ are the surface velocities of the journal and the shell, respectively. Note that there are two unknowns in Equation (5): $p$ and $\theta$. For $t=0$, ratio $\theta$ is prescribed by initial conditions (usually $\theta=1$ ) and Equation (5) is solved for $p$. If $p$ at any node drops below the value of saturation pressure $p_{c}$, then $p=p_{c}$ and Equation (5) is solved for $\theta$.

\section{Parameters of the analysed system}

An arrangement and dimensions of the analysed system are depicted in Figure 1. The system consists of the rotor supported on two journal bearings and a controller which controls the speed of the rotor. The controller is connected to the rotor with a bellows coupling with torsional stiffness $k_{r}=9.0 \cdot 10^{3}$ $\mathrm{N} \cdot \mathrm{m} \cdot \mathrm{rad}^{-1}$, axial stiffness $k_{a}=3.0 \cdot 10^{4} \mathrm{~N} \cdot \mathrm{m}^{-1}$ and lateral stiffness $k_{l}=3.2 \cdot 10^{5} \mathrm{~N} \cdot \mathrm{m}^{-1}$. The maximum speed of the rotor is $15,000 \mathrm{rpm}(250 \mathrm{~Hz})$. Properties of the journal bearings are summarised in Table 1 and their geometry is shown in Figure 2a. Note that bores for oil supply are located in the lower halves of the bearing shells.

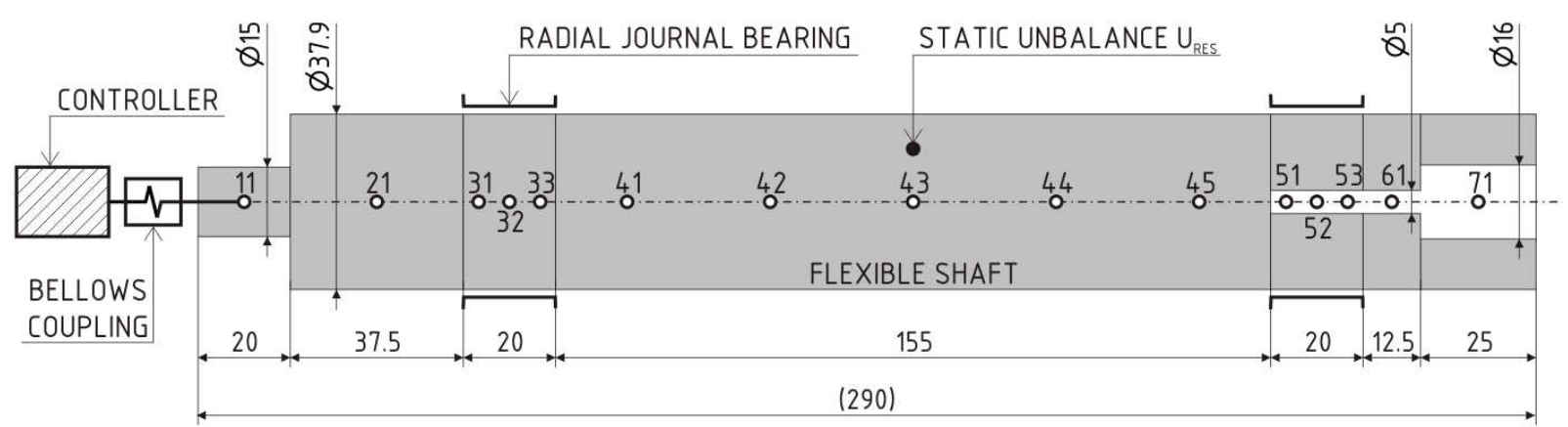

Fig. 1: Geometry and discretization of the rotor which is made of steel with the following material parameters: $E=205 \mathrm{MPa}, v=0.29, \rho=205 \mathrm{~kg} \cdot \mathrm{m}^{-3}$. The Rayleigh damping coefficients are $\alpha=5.027 \mathrm{~s}^{-1}$ and $\beta=1.273 \cdot 10^{-5} \mathrm{~s}$. 
Tab. 1: Nominal parameters of journal bearings. Position of the supply bore is depicted in Figure $2 a$.

\begin{tabular}{lcl||lcl}
\hline Parameter & Symbol & Value & Parameter & Unit & Value \\
\hline bearing diameter & $d$ & $38.0 \mathrm{~mm}$ & ambient pressure & $p_{a}$ & $1.00 \mathrm{bar}$ \\
\hline bearing length & $l$ & $20.0 \mathrm{~mm}$ & saturation pressure & $p_{c}$ & $0.98 \mathrm{bar}$ \\
\hline radial clearance & $c_{r}$ & $40.5 \mu \mathrm{m}$ & supply pressure & $p_{s}$ & $1.25 \mathrm{bar}$ \\
\hline lubricant viscosity & $\mu$ & $28.3 \mathrm{mPa} \cdot \mathrm{s}$ & supply bore diameter & $d_{b}$ & $5.00 \mathrm{~m}$ \\
\hline number of grid points & & $121 \times 21$ & supply bore position & $\phi_{b}$ & $180.0^{\circ}$ \\
\hline
\end{tabular}

\section{Results}

In order to understand dynamic properties of the rotor, the Campbell diagram was constructed from numerically evaluated values of complex eigenfrequencies. Hydrodynamic forces were linearized employing a method introduced in (Krämer, 2006) and the rotor damping was neglected $(\alpha=0, \beta=0)$. The resulting diagram (Figure $2 b$ ) suggests that one of the cylindrical rigid modes with natural frequency at half of the rotor speed goes unstable at $107.5 \mathrm{~Hz}$. The first lateral bending mode is located at 2,150 Hz. Therefore, the rotor can be considered rigid.

The steady-state response of the perfectly balanced rotor was simulated for frequency of rotation $f_{r}$ from range $f_{r} \in\langle 100 ; 250\rangle \mathrm{Hz}$. Equations of motion (1) - (4) were integrated numerically employing a backward differentiation method. The response was analysed in interval $t \in\langle 1 ; 2\rangle$ and is depicted in Figure 2c. A high pass-filter with cutoff frequency $3.5 \mathrm{~Hz}$ and the Hann window were applied and then the response was transformed into the frequency domain using FFT. A sub-synchronous component which

a)

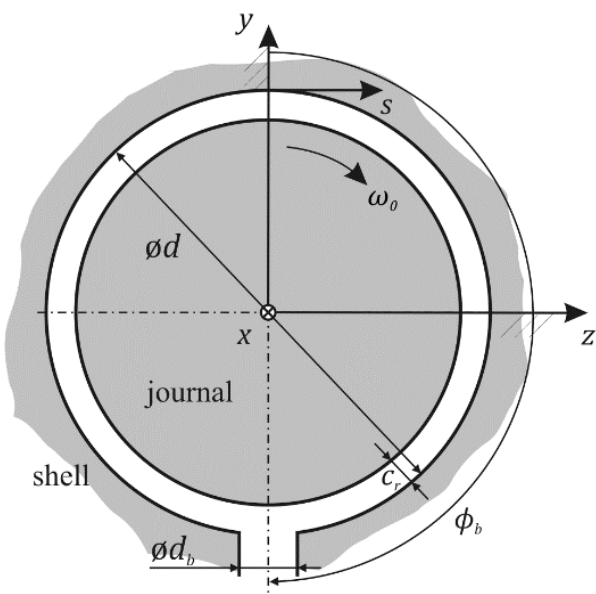

c)

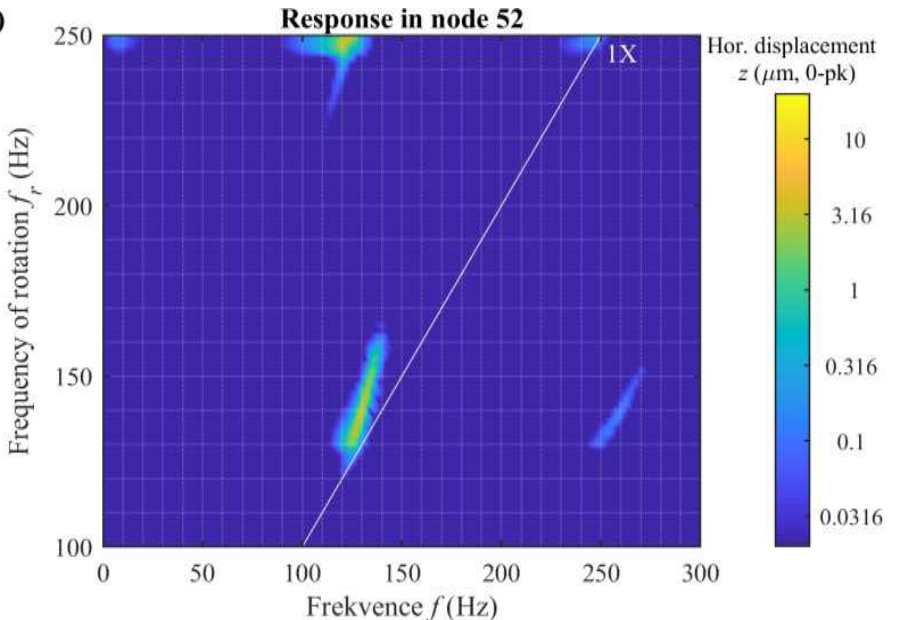

b)

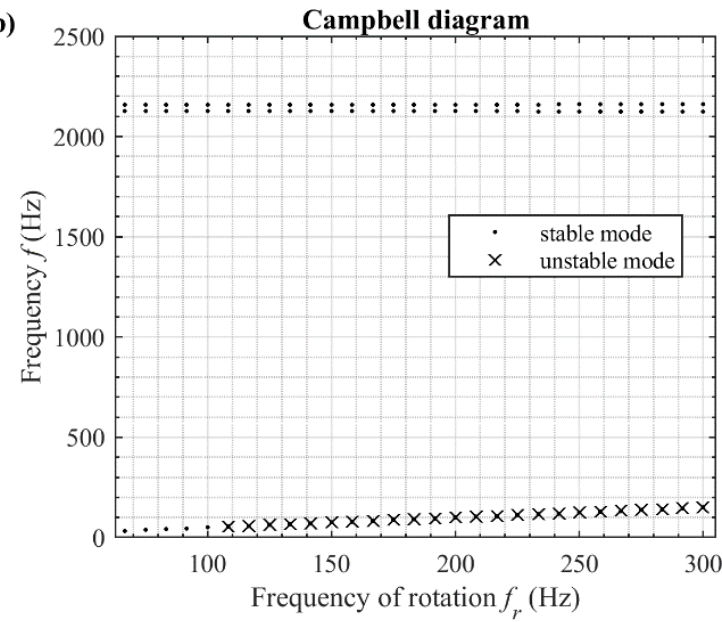

d)

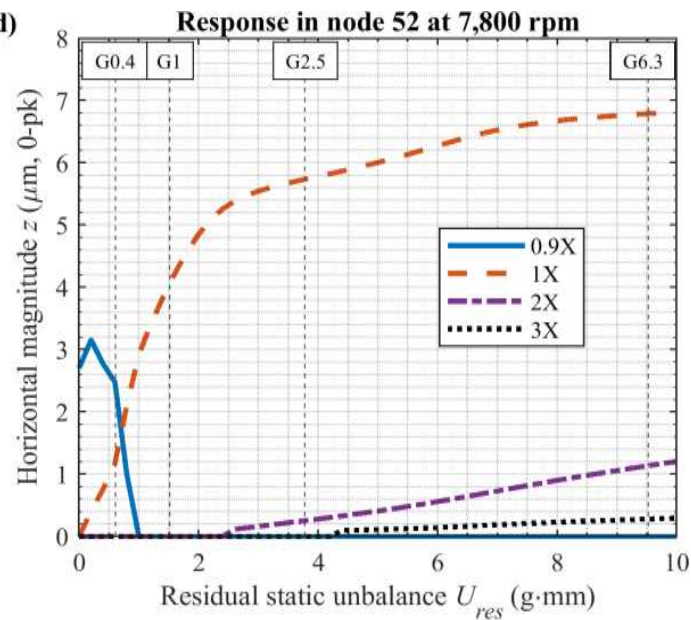

Fig. 2: (a) Geometry of the bearing. (b) Campbell diagram for the rotor. (c) Steady-state response of the perfectly balanced rotor. (d) Sensitivity of the response components on the static unbalance. 
a)

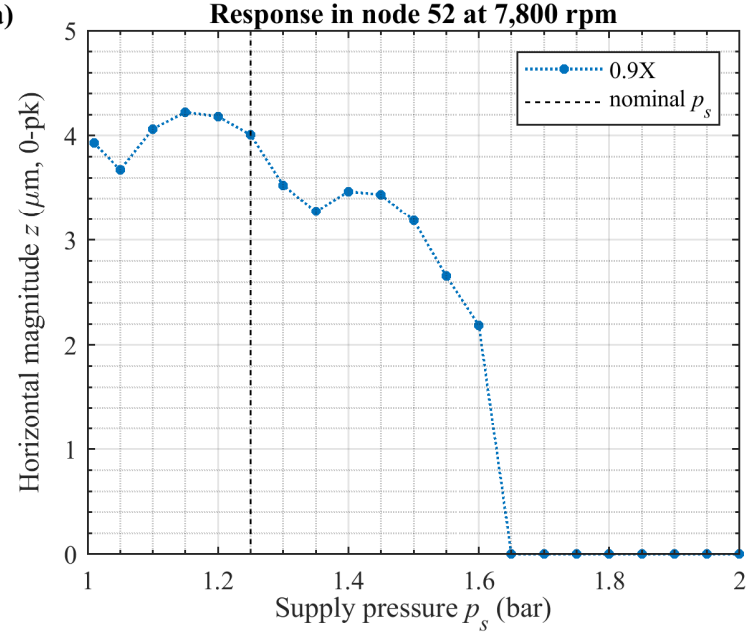

b)

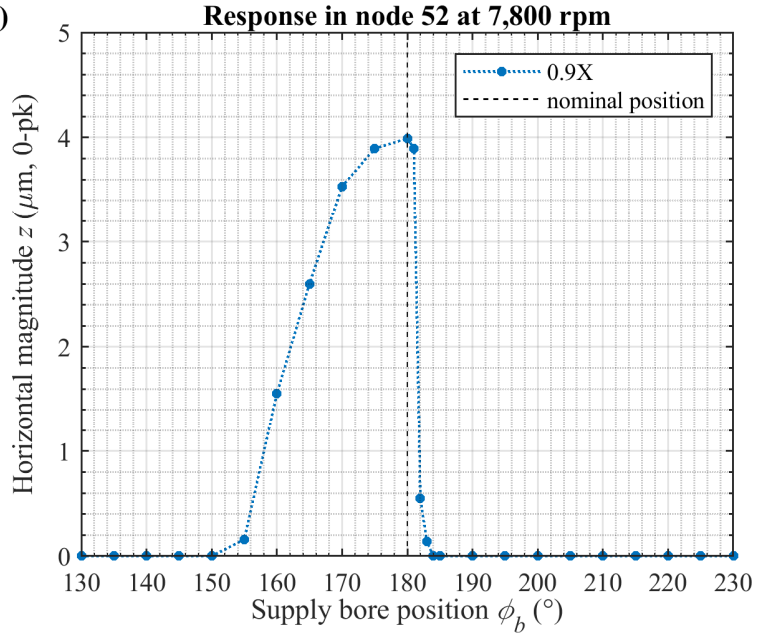

Fig. 3: Sensitivity of component $0.9 \mathrm{X}$ on (a) the supply pressure and on (b) the position of the bore.

is further referred to as $0.9 \mathrm{X}$ appears at $f_{r}=130 \mathrm{~Hz}$ and disappears at $f_{r} \approx 160 \mathrm{~Hz}$. The fluid-induced instability develops at $f_{r}=245 \mathrm{~Hz}$ at the frequency which is $0.48 \times f_{r}$.

Component $0.9 \mathrm{X}$ is presumably caused by parameters of oil supply. Figures $2 \mathrm{~d}$ and $3 \mathrm{a}$ illustrate that this sub-synchronous component is present only if the rotor is well-balanced and if the bearings are poorly lubricated. Figure 3a suggests that component $0.9 \mathrm{X}$ appears if the supply pressure is $1.6 \mathrm{bar}$ at the most. The appearance of $0.9 \mathrm{X}$ component is sensitive on the position of the supply bore. The bore has to be located within the interval $\phi_{b} \in\langle 155 ; 183\rangle$ degrees (Figure $3 b$ ). Angle $\phi_{b}$ is depicted in Figure 2a.

\section{Conclusions}

An occurrence of sub-synchronous vibrations at the frequency which is ca. 0.9 to the shaft speed is reported in this paper. The reported oscillations can occur only if a rotor is well balanced (balance quality grade G1 or lower per ISO 21940-1), and if it is radially supported on poorly lubricated journal bearings whose supply bores are located in the lower half of the bearings. These vibrations are stable and cover roughly $10 \%$ of the bearing clearance and, therefore, they are not dangerous. However, such vibrations might be undesirable in precise machinery.

\section{Acknowledgement}

The work has been supported by the project of the Czech Science Foundation No. 17-15915S "Nonlinear dynamics of rotating systems considering fluid film instabilities with the emphasis on local effects".

Simulations were performed in the AVL Excite software which is available in the framework of the University Partnership Program of AVL List GmbH and its usage is greatly acknowledged.

\section{References}

Bently, D. E. and Hatch, Ch. T. (2003) Fundamentals of Rotating Machinery Diagnostics. ASME Press, New York. DeCamillo, S. (2006) Current issues regarding unusual conditions in high-speed turbomachinery. In: 5th EDF \& LMS Poitiers Workshop: Bearing Behavior Under Unusual Operating Conditions. EDF, Paris, pp A.1-A.10.

Krämer, E. (1993). Dynamics of Rotors and Foundations. Springer-Verlag, Berlin Heidelberg.

Lorenz, N., Offner, G. and Knaus, O. (2017) Thermal analysis of hydrodynamic lubricated journal bearings in internal combustion engines. Proceedings of the Institution of Mechanical Engineers, Part K: Journal of Multibody Dynamics, 231, 3, pp 406-419.

Muszynska, A. (2005) Rotordynamics. CRC Press, Boca Raton.

Offner, G., Eizenberger, T. and Priebsch, H. H. (2006) Separation of reference motions and elastic deformations in an elastic multi-body system. Proceedings of the Institution of Mechanical Engineers, Part K: Journal of Multibody Dynamics, 220, 1, pp 63-75.

Someya, T. (1989) Journal-Bearing Databook. Springer, Berlin. 